\title{
VIRTUAL RESTORATION OF ARTWORKS USING ENTROPY-BASED COLOR IMAGE FILTERING SCHEMES
}

\author{
Rastislav Lukac ${ }^{1}$, Konstantinos N. Plataniotis ${ }^{1}$, Bogdan Smolka ${ }^{2}$ * \\ ${ }^{1}$ The Edward S. Rogers Sr. Department ECE \\ University of Toronto, 10 King's College Road \\ Toronto, M5S $3 G 4$ Ontario, Canada \\ lukacr@ieee.org, kostas@dsp.utoronto.ca \\ ${ }^{2}$ Polish-Japanese Institute of Information Technology, \\ Koszykowa 86 Str, 02-008 Warsaw, Poland \\ bsmolka@ia.polsl.gliwice.pl
}

\begin{abstract}
This paper presents a family of adaptive color image filters based on robust order-statistics and local contrast entropy control. The proposed filtering class is fully adaptive and free of any optimization procedure. Due to the adaptive nature of the algorithms and excellent balance between noise-removing and detailpreserving characteristics, it is desirable to use this technique as a pre-processing tool in practical color image processing applications such as virtual restoration of artworks.
\end{abstract}

Keywords: Color image processing; noise removal; virtual restoration of artworks.

\section{INTRODUCTION}

Digital artwork databases are created in order to preserve cultural heritage for the next generations ${ }^{1,2}$. Original artworks are documented by non-contact camera systems or laser scanner arrays, which gather accurately the corresponding digital data. However, digital artworks usually contain a number of impairments caused by ${ }^{3-5}:$ i) the changes in microclimatic conditions of original artworks resulting in varying color information, ii) scanning of granulated surfaces of artworks resulting in strong and discrete changes in the intensity,

*This research has been supported by a grant No PJ/B/01/2004 from the Polish - Japanese Institute of Information Technology. 
and iii) grime encrusting and natural defects and cracks present in the artworks. As a result, the digitized artworks suffer from color artifacts and image noise which inhibit correct recognition of the visual information ${ }^{5}$.

It is well-known that noise filtering can be used to enhance the perceptual quality of the images ${ }^{6}$. To improve visual appearance of the digitized artworks (e.g. for virtual restoration and digital interpretation purposes), the adaptive color image filters can be utilized ${ }^{5}$. Moreover, it also improves the performance of subsequent processing steps (e.g. image retrieval and object segmentation) utilized in digital archives.

\section{VECTOR MEDIAN FILTER}

Let us consider, a $K_{1} \times K_{2}$ color image $\mathrm{x}: Z^{2} \rightarrow Z^{3}$ representing a two-dimensional matrix of three-componentsamples $\mathbf{x}_{i}=\left[x_{i 1}, x_{i 2}, x_{i 3}\right]$, with $i=1,2, \ldots, K_{1} K_{2}$ and $x_{i 1}$ denoting the $\mathrm{R}$ component, $x_{i 2}$ denoting the $\mathrm{G}$ component and $x_{i 3}$ indicating the $\mathrm{B}$ component. Thus, each color pixel $\mathbf{x}_{i}$ is considered as a 3-dimensional vector in the RGB color space.

As described above, noise introduced into digitized artworks is present in forms of artifacts significantly deviating from neighboring pixels. This results in color distortion and impulsive nature of the noise corruption to which the human visual system is very sensitive. To remove outliers present on the color data, the most popular color filtering techniques, such as the vector median filter $(\mathrm{VMF})^{7}$, are operating on some type of sliding (moving, running) window $W=\left\{\mathbf{x}_{i}\right.$; for $\left.i=1,2, \ldots, N\right\}$ of a finite odd size $N$ which usually affects one image sample (mostly the window center $\mathbf{x}_{(N+1) / 2}$ ) at time, changing its value by some function of a local neighborhood area $\left\{\mathbf{x}_{1}, \mathbf{x}_{2}, . ., \mathbf{x}_{N}\right\}$. This window operator slides over the image to affect individually all image pixels.

The output of the VMF scheme is the sample $\mathbf{x}_{(1)} \in W$ minimizing the distance to other samples inside $W$ :

$$
\min \underset{\mathbf{x}_{(1)} \in W}{\arg } \sum_{i=1}^{N}\left\|\mathbf{x}_{(1)}-\mathbf{x}_{i}\right\|_{2}
$$

where $\left\|\mathbf{x}_{(1)}-\mathbf{x}_{i}\right\|_{2}$ denotes the Euclidean distance between two color vectors $\mathbf{x}_{i}$ and $\mathbf{x}_{j}$.

The VMF has the robust noise attenuation capability. However, the drawback is its low signal-detail preserving capability. To avoid excessive smoothing resulting in image blurring and elimination of fine image structures, switching filtering schemes are widely used. Vector filters introduced in ${ }^{8,9}$ employ local contrast entropy control to obtain the balance between smoothing and detail-preserving characteristics. 


\section{LOCAL CONTRAST ENTROPY BASED VECTOR FILTERS}

Assuming the input color vectors $\mathbf{x}_{1}, \mathbf{x}_{2}, . ., \mathbf{x}_{N}$ within $W$, the normalized deviation from the mean is given as follows ${ }^{5,8}$ :

$$
P_{i}=\frac{\left\|\mathbf{x}_{i}-\overline{\mathbf{x}}\right\|_{2}}{\sum_{j=1}^{N}\left\|\mathbf{x}_{j}-\overline{\mathbf{x}}\right\|_{2}}, \text { for } i=1,2, \ldots, N
$$

where $\overline{\mathbf{x}}$ is the mean of the input vectors $\mathbf{x}_{1}, \mathbf{x}_{2}, . ., \mathbf{x}_{N}$.

Applying the entropy concept to $\mathbf{x}_{1}, \mathbf{x}_{2}, . ., \mathbf{x}_{N}$, the input samples contribute to the cumulated entropy defined by $H=-\sum_{i=1}^{N} P_{i} \log P_{i}$, where $P_{i}$ is the normalized local contrast (2) associated with the input vector sample $\mathbf{x}_{i}$ and $N$ is the window size. In terms of the entropy concept, each input sample $\mathbf{x}_{i}$, for $i=1,2, \ldots, N$, is also associated with the local contrast entropy given by $H_{i}=$ $-P_{i} \log P_{i}$. The cumulated entropy and local contrast entropy definitions can be employed in the fully adaptive VMF filtering scheme ${ }^{5}$. The adaptive control of the VMF smoothing mode and identity operation is obtained by comparing the adaptive threshold $\beta_{i}$ with the normalized local contrast $P_{i}$ of (2). Because each $P_{i}$, for $i=1,2, \ldots, N$, is always constrained to be a value between 0 and 1 , the adaptive threshold of our approach should be re-scaled to the same interval. Therefore, the local contrast entropy $H_{i}$ and the cumulated entropy $H$ of the input color vectors $\mathbf{x}_{1}, \mathbf{x}_{2}, . ., \mathbf{x}_{N}$ are used as follows ${ }^{8}$ :

$$
\beta_{i}=\frac{H_{i}}{H}=\frac{P_{i} \log P_{i}}{\sum_{j=1}^{N} P_{j} \log P_{j}}
$$

The switching rule employs the comparison of the local contrast probability and the adaptive threshold, both corresponding to the color vector positioned in the center of filtering window. The adaptive entropy vector median filter (AEVMF) output $\mathbf{y}$ is defined as follows ${ }^{5,8}$ :

$$
\mathbf{y}= \begin{cases}\mathbf{x}_{(1)} & \text { for } P_{(N+1) / 2} \geq \beta_{(N+1) / 2} \\ \mathbf{x}_{(N+1) / 2} & \text { otherwise }\end{cases}
$$

where $\mathbf{x}_{(1)}$ is the lowest ranked vector equivalent to the standard VMF output, $\beta_{(N+1) / 2}$ is the adaptive threshold and $P_{(N+1) / 2}$ denotes the local contrast probability associated with $\mathbf{x}_{(N+1) / 2}$.

It is clear that the decision operation $P_{i} \geq \beta_{i}$ can be related to each input color vector $\mathbf{x}_{i}$, for $i=1,2, . ., N$. As a result we achieve a noise-free set of image samples $W^{\prime}=\left\{\mathbf{x}_{1}^{\prime}, \mathbf{x}_{2}^{\prime}, \ldots, \mathbf{x}_{r}^{\prime}\right\}$, for $r \leq N$. If $\mathbf{x}_{(N+1) / 2} \in W^{\prime}$, then no other operation is necessary and $\mathbf{x}_{(N+1) / 2}$ remains unchanged. However, if $\mathbf{x}_{(N+1) / 2}$ is noisy, then it is desired to constitute the filter estimate only from the noise-free samples of $W^{\prime}$. Therefore, this technique can increase the accuracy of $(4)^{5}$. 
Replacing the sample mean $\overline{\mathbf{x}}$ of (2) with the lowest ranked vector $\mathbf{x}_{(1)}$ constitutes the ranking based entropy vector median filter. This filtering technique uses the same switching rule of (4), however, all required quantities such as the normalized local contrast $P_{i}$ and the adaptive parameter $\beta_{i}$ are calculated using the following expression ${ }^{5}$ :

$$
P_{i}=\frac{\left\|\mathbf{x}_{i}-\mathbf{x}_{(1)}\right\|_{2}}{\sum_{j=1}^{N}\left\|\mathbf{x}_{j}-\mathbf{x}_{(1)}\right\|_{2}}, \text { for } i=1,2, \ldots, N
$$

where $\mathbf{x}_{(1)}$ is the lowest ranked sample equivalent to the standard VMF output. Applying (5), the proposed adaptive framework also utilizes the VMF output in the switching filter stage. This increases robustness of the method, ${ }^{5}$ since the VMF operator is more useful for image environments impulsive in nature than the sample mean. Note that various filtering schemes constructed within the presented framework can be achieved by considering different distance measures in $(2)^{9}$.

\section{APPLICATION TO DIGITIZED ARTWORKS}

The performance of the presented adaptive VMFs is tested using digitized artworks shown in Fig.1a and Fig.2a. Since we deal with a real problem, the original signal is unavailable. Therefore, standard, objective image quality measures based on the difference in the statistical distributions of the pixel values cannot be utilized.

The proposed framework is compared, in terms of the visual quality, with widely used color image filters, such as componentwise median filter (MF), ${ }^{6}$ $\mathrm{VMF}^{7}$ and basic vector directional filter (BVDF) ${ }^{6}$.

Fig.1 demonstrates the filter efficiency when applied to the digitized artwork. Standard filtering schemes (MF, VMF and BVDF) remove image noise, however their strong smoothing capability results in significantly blurred image details (Fig.1b-d). Fig.1e demonstrates that the proposed AEVMF scheme achieves the best trade-off between noise attenuation and signal-detail preservation. Similar conclusions can be drawn through visual inspection of the results shown in Fig.2.

\section{CONCLUSION}

The paper presented an adaptive VMF filtering framework. The performance of the framework has been tested using the digitized artwork images. The use of the local contrast entropy switching concept significantly improves the signal-detail preservation capability of the conventional VMF technique. The switching VMF schemes remove discrete color artifacts and pixels significantly deviated from their neighbors and excellently preserve color/structural 
(a)

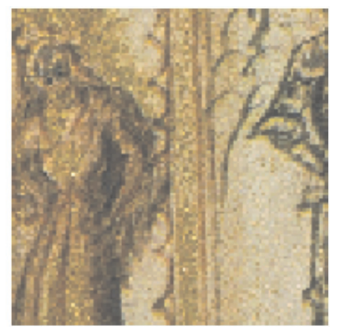

(b)

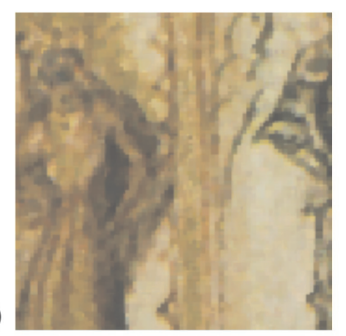

(c)

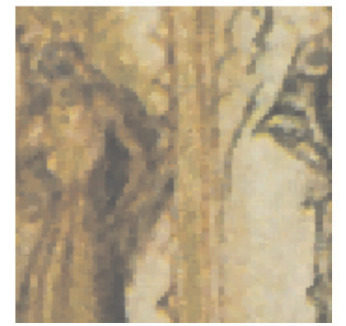

(d)

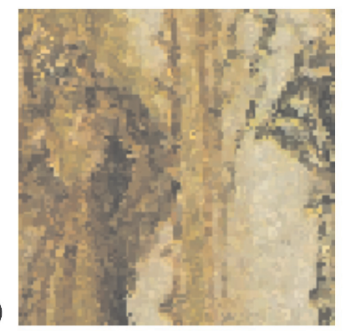

(e)

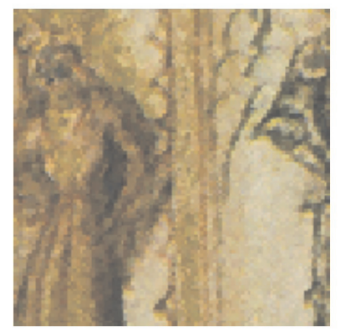

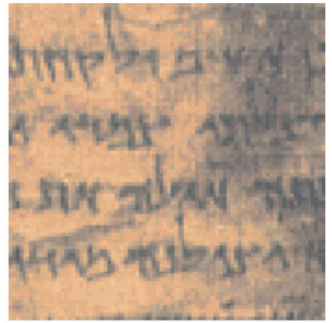
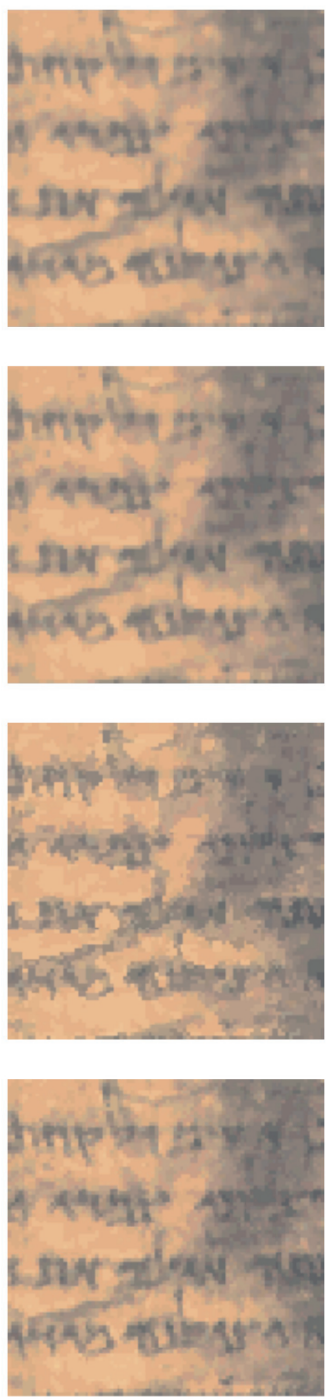
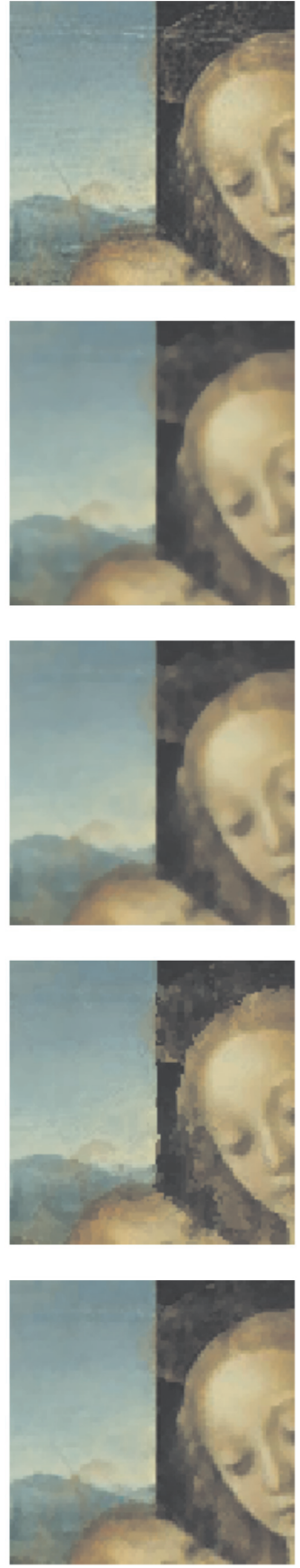

Figure 1. Zoomed parts of the obtained results: (a) input image, (b) MF output, (c) VMF output, (d) BVDF output, (e) AEVMF output. 


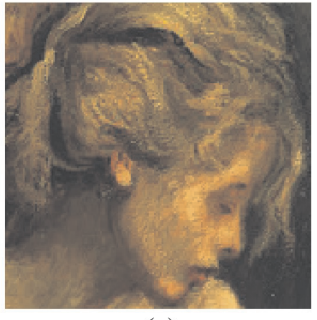

(a)

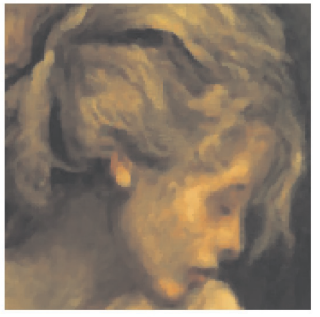

(b)

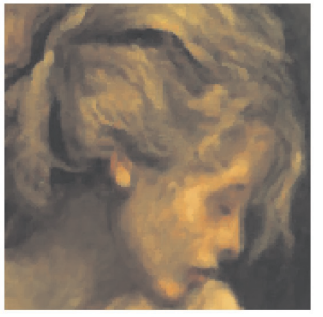

(c)

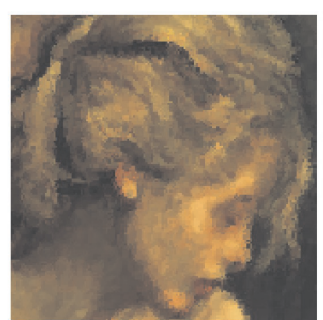

(d)

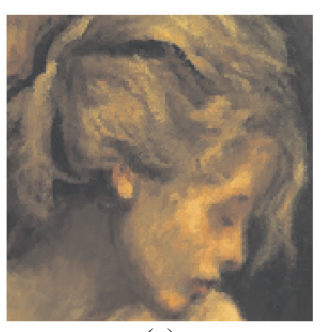

(e)

Figure 2. Zoomed parts of the obtained results: (a) input image, (b) MF output, (c) VMF output, (d) BVDF output, (e) AEVMF output.

information of digitized artworks. At the same time, they are sufficiently robust and provide better results than the widely used filtering schemes.

\section{REFERENCES}

1. A. C. Addison, Emerging trends in virtual heritage, IEEE Multimedia, 7, 22-25, April-June 2000.

2. J. Y. Zheng, Virtual recovery and exhibition of heritage, IEEE Multimedia, 7, 31-34, AprilJune 2000.

3. M. Barni, F. Bartolini, V. Cappelini, Image processing for virtual restoration of artworks, IEEE Multimedia, 7, 34-37, April-June 2000.

4. X. Li, D. Lu, Y. Pan, Color restoration and image retrieval for Donhuang fresco preservation, IEEE Multimedia, 7, 38-42, April-June 2000.

5. R. Lukac, B. Smolka, K. N. Plataniotis, Adaptive color image filter for application in virtual restoration of artworks, IEEE Transactions on Multimedia, submitted.

6. K. N. Plataniotis, A. N. Venetsanopoulos, Color Image Processing and Applications. Springer Verlag, Berlin, 2000.

7. J. Astola, P. Haavisto, Y. Neuvo, Vector median filters, Proceedings of the IEEE, 78, 678-689, April 1990.

8. R. Lukac, B. Smolka, K. N. Plataniotis, A. N. Venetsanopoulos, Entropy vector median filter. Proc. IbPRIA'03 in Puert de Andtrax, Spain, 1117-1125, June 2003.

9. R. Lukac, B. Smolka, K. N. Plataniotis, A. N. Venetsanopoulos, Generalized entropy vector filtering, Proc. EC-VIP-MC'3 in Zagreb, Croatia, 239-244, July 2003. 